\title{
Factores determinantes que permiten establecer tipologías de profesorado en el contexto de la innovación tecnológica educativa.
}

\section{Determining factors that make it possible to establish typologies of teachers in the context of technological innovation in education.}

\author{
Byron Geovanny Hidalgo-Cajo \\ Universidad Nacional de Chimborazo (UNACH). Riobamba, Ecuador \\ bhidalgo@unach.edu.ec \\ Mercé Gisbert-Cervera \\ Universitat Rovira i Virgili (URV). Tarragona, España \\ merce.gisbert@urv.cat
}

\section{Resumen}

La figura del docente tiene un papel fundamental en la integración de las Tecnologías digitales (TD) en el proceso enseñanza aprendizaje, desde esta óptica se han elaborado diversos estudios con el objetivo de definir cuáles son las variables y/o factores más influyentes en la adopción, uso e integración de las TD desde la perspectiva del profesorado, de los cuales muy pocos llegan a concluir con una definición clara. Desde este punto de vista el estudio tiene como objetivo diseñar un modelo instruccional teórico explicativo de los diferentes perfiles del profesorado que aúne todas las variables y factores que pueden intervenir en la integración de las tecnologías digitales en el ámbito educativo, centrándose en las necesidades, tipos y características del profesorado. A partir del análisis de la literatura y estudios pragmáticos previos se han identificado diferentes variables que influyen en la integración de las TD y que han permitido diseñar un modelo de los perfiles del profesorado desde la perspectiva de la práctica docente, que determinan las etapas de adopción, aplicando paradigmas contextuales. El estudio concluye que las variables determinantes para la apropiación de las TD desde el contexto del profesorado son la Actitud y la Autoeficacia, a las que se suman los factores: Utilidad de uso, Propiedad, acceso a las TD, Formación docente, Facilidad de uso, Importancia, presión social e Interés ante las TD. Estas variables y factores dan lugar a crear un modelo instruccional de aproximación teórica y explicativa de los cinco perfiles de profesorado con respecto a la adopción, uso e integración de las TD en el proceso educativo, las cuales hemos etiquetado como: "resistente", "confuso" "adoptante", "persuadido" e "innovador".

Palabras clave: Competencia digital; Perfiles Docentes; Tecnología digital; Tecnología educativa; Usos educativos de la tecnología.

\begin{abstract}
The figure of the teacher plays a fundamental role in the integration of digital technologies (TD) in the teaching-learning process. From this perspective, several studies have been carried out with the aim of defining the most influential variables and/or factors in the adoption, use and integration of TD from the teacher's perspective, of which very few conclude with a clear definition. From this point of view, the study aims to design an explanatory theoretical instructional model of the different teacher profiles that brings together all the variables and factors that can
\end{abstract}


intervene in the integration of digital technologies in the educational environment, focusing on the needs, types and characteristics of teachers. Based on the analysis of the literature and previous pragmatic studies, different variables have been identified that influence the integration of TD and that have allowed the design of a model of teacher profiles from the perspective of teaching practice, which determine the stages of adoption, applying contextual paradigms. The study concludes that the determining variables for the appropriation of TD from the context of teachers are Attitude and Self-efficacy, to which are added the factors: Usefulness of use, Ownership, access to TD, Teacher training, Ease of use, Importance, social pressure and Interest in TDs. These variables and factors lead to the creation of an instructional model of theoretical and explanatory approximation of the five teacher profiles with respect to the adoption, use and integration of TD in the educational process, which we have labelled as: "resistant", "confused", "adopter", "persuaded" and "innovator".

Keywords: Digital competence; Teacher profiles; Digital technology; Educational technology; Educational uses of technology.

\section{Introducción}

La inserción de las tecnologías digitales (TD) en las instituciones educativas constituye un desafío cada vez más apremiante en la educación. Desde este contexto el profesorado es uno de los actores principales en todo aquello relativo al uso, adopción e integración de las TD en el proceso enseñanza aprendizaje, que cuando se implementa con calidad trae consigo beneficios desde la perspectiva de las estrategias didácticas, y tecnológicas contribuyendo así a la mejora del proceso educativo.

Sin embargo, la adopción, uso e integración de la tecnología en el contexto educativo no debe determinarse, solamente en términos de la cantidad de aparatos tecnológicos que se emplean en el aula, y la utilización de estas como medio de apoyo en las actividades educativas tradicionales, sino que es necesario asociar lo instrumentista con lo pedagógico y didáctico en este proceso. Por consiguiente, la tecnología educativa hace referencia a la aplicación de ideas de diversas fuentes para crear los mejores entornos de aprendizaje por medio de la integración tecno pedagógica, que conlleve a la transformación del currículo o plan de estudios (Goh y Sigala, 2020), dicho de otra manera, que no solo se complemente a los procesos de enseñanza aprendizaje, sino que además los redefina, generando procesos innovadores.

La tecnología ha permitido transformar el proceso enseñanza aprendizaje, ya que su aplicación en las diferentes etapas de la práctica formativa ha logrado innovar el proceso educativo con énfasis en el trabajo más interactivo, colaborativo y cooperativo (Hidalgo Cajo et al., 2017), desde esta perspectiva diversos estudios corroboran y demuestran que las TD pueden ser utilizadas para mejorar el proceso de enseñanza por parte del profesorado y favorecer el rendimiento académico de los estudiantes (Davies et al., 2017; Sung et al., 2016; Li y Tsai, 2017; Kirkwood y Price, 2014), sin embargo las dificultades que el profesorado tiene que afrontar en la adopción y uso de las TD son: 1. de acceso (insuficiente equipamiento); 2. pedagógicos (insuficiente nivel de competencias, falta de apoyo técnico-educativo, carencia de materiales didácticos, ausencia de modelos para la educación digital); y 3. actitudinales (resistencia del profesorado y/o familias, bajo interés del docente, ausencia de percepción de beneficios, no se identifica como una meta del centro educativo) (European Commission, 2019).

Factores determinantes que permiten establecer tipologías del profesorado en el contexto de la innovación tecnológica educativa. B. Hidalgo-Cajo y M. Gisbert-Cervera. Página 2 de 23 
Por otra parte, las TD en el campo educativo han generado altas expectativas, tales como herramientas de apoyo a la presencialidad, así como un medio para fortalecer las alternativas de formación en las modalidades no presenciales. A partir de la incorporación de las TD se han implementado acciones de formación y evaluación que permiten valorar o avalar el uso apropiado dentro de las distintas áreas educativas como la docente, curricular, administrativa, tutorial, entre otras (Pérez y Andrade, 2020).

A su vez se debe considerar al profesorado como el sujeto principal en la decisión libre y voluntaria del proceso de adopción y uso de las TD. Este proceso debe unirse a un análisis de las características individuales de los docentes, unido a la consideración de factores de tipo personal y contextual en el proceso enseñanza aprendizaje que están sujetos a cambios dinámicos que pueden ser reforzados o contrarrestados mediante acciones y/o técnicas orientadas por las instituciones universitarias (Martin-Garcia et al., 2014).

Gil-Flores et al. (2017) manifiestan que la adopción y uso e integración de las TD por parte del profesorado depende de múltiples variables, pero entre ellas no están las demográficas (edad, sexo, experiencia docente) puesto que son irrelevantes para explicar el uso de las TD (ValdésCuervo et al., 2009; Garcia et al., 2020). Por otra parte, evidencian que la necesidad percibida de formación en TD es la variable más significativa para la integración de las tecnologías en la práctica docente, de la misma manera precisan que la probabilidad de incremento del uso de las $\mathrm{TD}$, se manifiesta de forma más clara si el docente posee un enfoque constructivista del proceso de enseñanza-aprendizaje o si existe una cultura de colaboración entre docentes.

\subsection{Objetivo de la Investigación}

La finalidad de la investigación es realizar una revisión sistemática de la literatura que identifique las variables y factores determinantes en función a la adopción, uso e integración de las TD desde el contexto del profesorado, con el fin de diseñar un modelo instruccional teórico explicativo de los diferentes perfiles de profesorado.

\section{Metodología}

\subsection{Revisión Sistemática de la Literatura.}

Con el fin de cumplir con el objetivo de la investigación se realiza una revisión sistemática de la literatura (SLR), que es el proceso de construcción teórica cuyo propósito es revisar los documentos más relevantes en el campo de estudio, independientemente del acceso a cualquier dato primario (Okoli y Schabram, 2010), el cual es llevado a cabo de forma sistemática, rigurosa y estandarizada, permitiendo la meta-revisión en etapas sucesivas. El corpus bibliográfico final proviene de una recopilación de documentos sin ninguna restricción temporal, así como también no se restringe la ubicación de los descriptores ya sea en el título, resumen, palabras clave o cuerpo del texto, los documentos seleccionados cuentan con criterios de calidad, pertinencia y relevancia, y se centra en identificar las variables y factores determinantes en función a la adopción y uso de las TD desde el contexto del profesorado, con el fin de diseñar un modelo teórico explicativo de los diferentes perfiles del profesorado, para la búsqueda de la literatura se utilizó operadores booleanos tanto en inglés como en español, y se consideraron las bases de datos científicas SCOPUS y Web of Science (WoS).

\subsection{Criterios de inclusión y proceso de selección.}

Durante la primera fase de identificación se logró recoger el mayor número de documentos posibles, especialmente teniendo en cuenta la necesidad de ofrecer una visión general del estudio. El motor de búsqueda proporciono 980 documentos, 720 en SCOPUS y 260 en WoS, utilizando las siguientes cadenas de búsqueda:

Factores determinantes que permiten establecer tipologías del profesorado en el contexto de la innovación tecnológica educativa. B. Hidalgo-Cajo y M. Gisbert-Cervera. Página 3 de 23 
WoS

$\mathrm{TS}=(($ variable* OR Factor*) AND (adoption* OR use* OR integration*) AND ("digital technologies") AND (teacher* OR Professor*))

TS = ((“teacher profiles") OR (“teacher typologies") AND (adoption* OR Use* OR integration*) AND ("digital technologies"))

\section{SCOPUS}

TITLE-ABS-KEY = (variables OR factors) AND (adoption OR use OR integration) AND ("digital technologies") AND (teacher OR Professor)

TITLE-ABS-KEY = ("teacher profiles") OR ("teacher typologies") AND (adoption OR Use OR integration) AND ("digital technologies")

En esta fase los documentos seleccionados de las dos bases de datos científicas fueron exportados al software Parsifal que facilito la planificación de la revisión bibliográfica.

En la segunda fase o de eliminación, se procede a verificar si los documentos registrados se encuentran duplicados, el resultado de este procedimiento arrojo que 124 elementos están en esta situación mismos que se procedió a eliminar obteniendo 856 documentos seleccionados

En la tercera fase o de selección, se realiza la revisión de títulos y resúmenes de los documentos aplicando los criterios de inclusión y exclusión, en este punto son elegidos aquellos documentos que incluyan las siguientes características principales:

- Relacionadas con las variables y factores que influyen en la adopción, uso e integración de las TD por parte del profesorado.

- Relacionadas con los diferentes modelos de perfiles desde el contexto del profesorado en el proceso de adopción y uso de las TD.

De esta manera y mediante la revisión de los manuscritos, se obtuvo un corpus bibliográfico de 170 documentos.

En la cuarta fase o de inclusión, se procede a revisar y evaluar el texto completo del documento, y se ejecuta los criterios de calidad, pertinencia y relevancia mediante la aplicación de 10 preguntas que logran valorar el documento en función del objetivo del estudio, con tres opciones de respuesta: SI CUMPLE ( 1 punto), NO CUMPLE ( 0 punto) y parcialmente $(0,5$ puntos), como punto de corte para la inclusión de las publicaciones al estudios se estableció la valoración mínima de 7,5 puntos, lo que permitió tras la depuración bibliográfica la selección de 60 publicaciones, los mismos que cubren un periodo de tiempo que va desde el año 2000 hasta el 2021. Este proceso se representa en la Figura 1.

Factores determinantes que permiten establecer tipologías del profesorado en el contexto de la innovación tecnológica educativa. B. Hidalgo-Cajo y M. Gisbert-Cervera. Página 4 de 23 


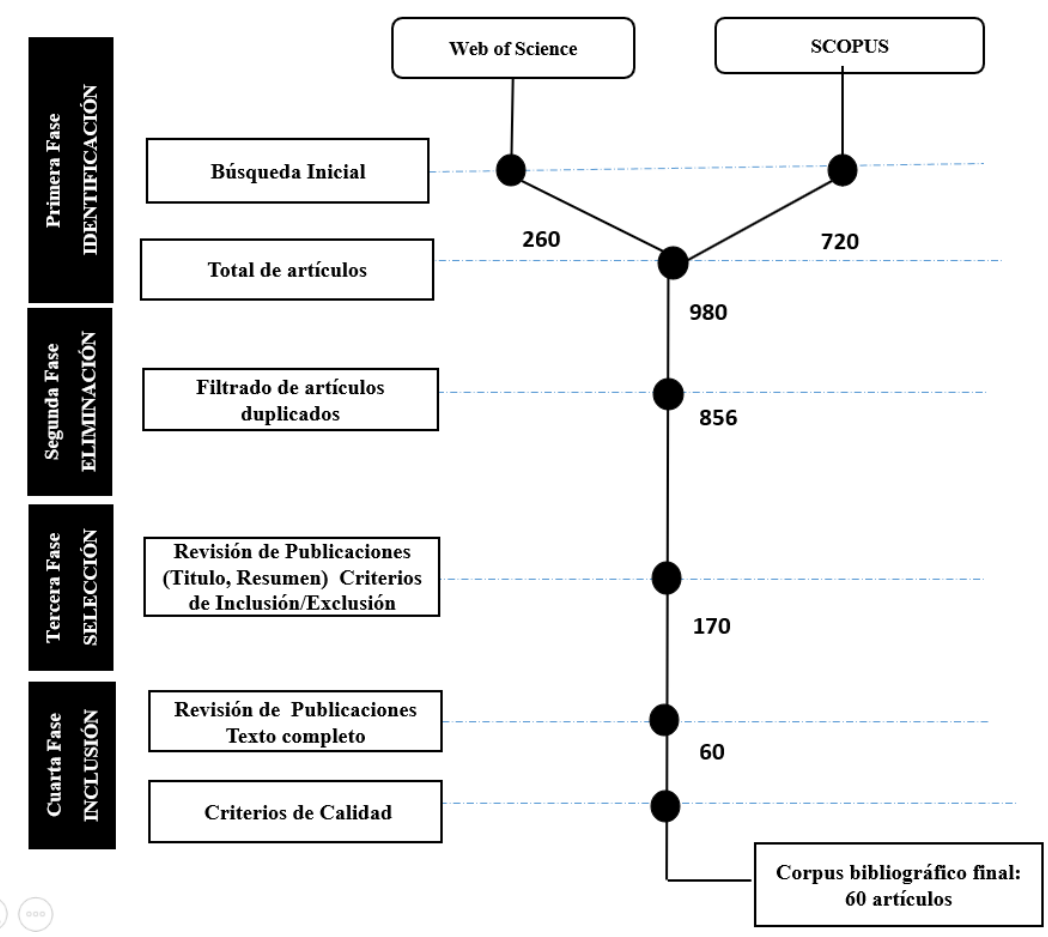

Figura 1.Proceso de creación de la base de datos documental

\section{Resultados}

Diversas investigaciones han evidenciado las variables que influyen el uso de las TD, es así que Hu et al. (2018) y Naik et al. (2020) consideran que la disponibilidad y acceso a equipamientos son variables que influyen en la adopción y uso de las TD. De la misma manera Christensen et al. (2018) y Wu et al. (2019) afirman que la capacitación del profesorado en TD tiene un papel importante a la hora de facilitar y apoyar la integración efectiva e innovadora de la tecnología en las instituciones educativas, siendo esta variable muy importante en la mejora de los sistemas educativos.

Desde el contexto del profesorado estudios como los de Sánchez-Mena et al. (2017) manifiestan que las variables utilidad y facilidad de uso percibido influyen de forma directa y positiva en la intención de los profesores de utilizar las TD. De la misma forma al analizar desde la perspectiva competencial Rolf et al. (2019), afirman que la competencia digital es una variable relevante en el proceso enseñanza aprendizaje mediada por el uso de las TD. A todo ello podemos añadir las conclusiones a las que llegan Njiku et al. (2019) cuando hacen referencia a la actitud del profesorado hacia las TD, siendo la utilidad, la confianza, la ansiedad y la posición del profesorado, las variables más representativas que determinan una actitud positiva hacia la integración de las TD.

Para Birisci \& Kul (2019) la variable autoeficacia es un indicador muy importante en la integración y uso de las TD en el proceso enseñanza aprendizaje desde la perspectiva del profesorado (Hall \& Trespalacios, 2019), estos estudios se alinean al de Tondeur et al. (2019) quienes afirman que existe una correlación positiva entre la autoeficacia y la actitud en la adopción y uso de la TD en el proceso educativo. Sin embargo algunos estudios (Tafazoli et al., 2019; Mercader, 2019; Hidalgo-Cajo et al., 2021; Muhaimin et al., 2020), afirman que las variables demográficas como la edad, genero, y nivel educativo no influyen significativamente en la actitud del uso de las TD. Como se puede apreciar diversos autores discrepan en las variables

Factores determinantes que permiten establecer tipologías del profesorado en el contexto de la innovación tecnológica educativa. B. Hidalgo-Cajo y M. Gisbert-Cervera. Página 5 de 23 
RED. Revista de Educación a Distancia. Núm. 69, Vol. 22. Artíc. 7, 30-Ene-2022

DOI: http://dx.doi.org/10.6018/red.488171

que consideran influyentes en la adopción, uso e integración de las TD sin tener una clara definición de las mismas.

En la Tabla 1 se presenta a modo de resumen y a partir de la revisión de la literatura, las diferentes variables consideradas más significativas con respecto al uso y la adopción de las TD por parte del profesorado

\section{Tabla 1.}

Variables determinantes para el uso de las TD desde el contexto el profesorado.

\begin{tabular}{|c|c|c|c|c|c|c|c|c|c|c|}
\hline \multirow[t]{2}{*}{ Autores } & \multicolumn{10}{|c|}{ Variables } \\
\hline & 1 & 2 & 3 & 4 & 5 & 6 & 7 & 8 & 9 & 10 \\
\hline (Holland \& Piper, 2016) & & & & & & & & & & \\
\hline (Tondeur, Pareja Roblin, et al., 2017) & & & & & & & & & & \\
\hline (Bervell \& Umar, 2017) & & & & & & & & & & \\
\hline (Admiraal et al., 2017) & & & & & & & & & & \\
\hline (Jo Tondeur et al., 2016) & & & & & & & & & & \\
\hline (Scherer \& Siddiq, 2015) & & & & & & & & & & \\
\hline (Siddiq et al., 2016) & & & & & & & & & & \\
\hline (Sang et al., 2012) & & & & & & & & & & \\
\hline (Tondeur et al., 2018) & & & & & & & & & & \\
\hline (Drossel et al., 2017) & & & & & & & & & & \\
\hline (Morales Arce, 2013) & & & & & & & & & & \\
\hline (Wen \& Hua, 2020) & & & & & & & & & & \\
\hline (Cózar et al., 2015) & & & & & & & & & & \\
\hline (Tadeu, 2020) & & & & & & & & & & \\
\hline (Trujillo-Torres et al., 2020) & & & & & & & & & & \\
\hline (Ocaña-Fernández et al., 2020) & & & & & & & & & & \\
\hline (Muhaimin et al., 2020) & & & & & & & & & & \\
\hline (Kisirkoi, 2015) & & & & & & & & & & \\
\hline (Simin \& Sani, 2015) & & & & & & & & & & \\
\hline (Babić et al., 2020) & & & & & & & & & & \\
\hline (Marín-Díaz et al., 2020) & & & & & & & & & & \\
\hline (Mosquera-González et al., 2021) & & & & & & & & & & \\
\hline (Ursavaş et al., 2019) & & & & & & & & & & \\
\hline (Njiku et al., 2019) & & & & & & & & & & \\
\hline (Hall \& Trespalacios, 2019) & & & & & & & & & & \\
\hline (Birisci \& Kul, 2019) & & & & & & & & & & \\
\hline (Martin-Garcia et al., 2014) & & & & & & & & & & \\
\hline (Sánchez-Mena et al., 2017) & & & & & & & & & & \\
\hline (Valdés-Cuervo et al., 2009) & & & & & & & & & & \\
\hline (Badia \& Iglesias, 2019) & & & & & & & & & & \\
\hline (Aguilar-Flores \& Chiang-Vega, 2020) & & & & & & & & & & \\
\hline$(\mathrm{Teo}, 2011)$ & & & & & & & & & & \\
\hline (Rodriguez et al., 2009) & & & & & & & & & & \\
\hline (Peinado et al., 2011) & & & & & & & & & & \\
\hline (Ertmer \& Ottenbreit-Leftwich, 2010) & & & & & & & & & & \\
\hline (Farjat-aguilar \& Barroso-tanoira, 2009) & & & & & & & & & & \\
\hline (Araiza \& Pedraza, 2019) & & & & & & & & & & \\
\hline (Zempoalteca Durán et al., 2017) & & & & & & & & & & \\
\hline (Pérez et al., 2016) & & & & & & & & & & \\
\hline (Fernández-Márquez et al., 2018) & & & & & & & & & & \\
\hline (Palos-Sanchez et al., 2019) & & & & & & & & & & \\
\hline (Morales, 2000) & & & & & & & & & & \\
\hline (Ertmer \& Ottenbreit-Leftwich, 2010) & & & & & & & & & & \\
\hline (Hixon \& Buckenmeyer, 2009) & & & & & & & & & & \\
\hline (Holmes et al., 2007) & & & & & & & & & & \\
\hline (Hassan \& Rahim Sajid, 2013) & & & & & & & & & & \\
\hline (Farjat-aguilar \& Barroso-tanoira, 2009) & & & & & & & & & & \\
\hline
\end{tabular}

Factores determinantes que permiten establecer tipologías del profesorado en el contexto de la innovación tecnológica educativa. B. Hidalgo-Cajo y M. Gisbert-Cervera. 
RED. Revista de Educación a Distancia. Núm. 69, Vol. 22. Artíc. 7, 30-Ene-2022

DOI: http://dx.doi.org/10.6018/red.488171

\begin{tabular}{|l|l|l|l|l|l|l|l|l|l|l|}
\hline (Goh \& Sigala, 2020) & & & & & & & & & & \\
\hline (Ndlovu et al., 2020) & & & & & & & & & & \\
\hline (Badia et al., 2016) & & & & & & & & & & \\
\hline (Petko, 2012) & & & & & & & & & & \\
\hline (Zempoalteca Durán et al., 2017) & & & & & & & & & & \\
\hline (Bin et al., 2020) & & & & & & & & & & \\
\hline (Selcen Guzey \& Roehrig, 2012) & & & & & & & & & & \\
\hline (Donnelly et al., 2011) & & & & & & & & & & \\
\hline (Mirzajani et al., 2015) & & & & & & & & & & \\
\hline
\end{tabular}

En el Grafico 1, se representa la frecuencia de las variables que han sido analizadas en las diferentes publicaciones. Se han desestimado aquella que tenían una frecuencia menor a cuatro.

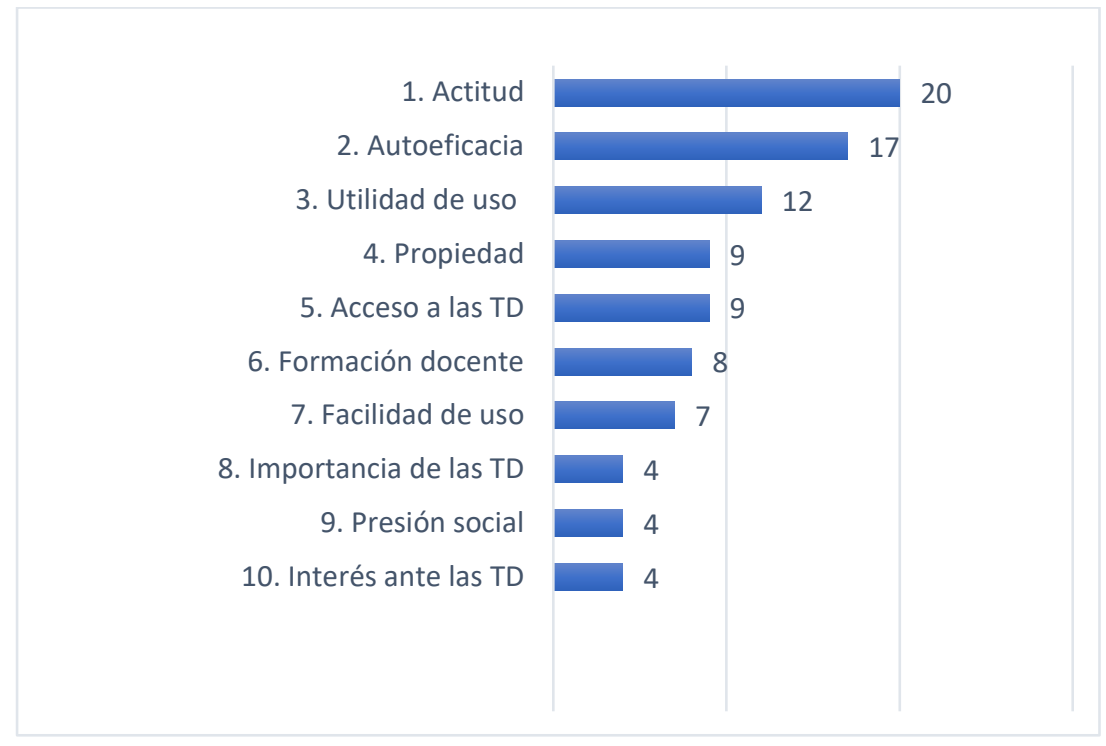

Gráfico 1. Variables determinantes para la adopción, uso e integración de las TD desde el contexto del profesorado.

El análisis de la literatura nos ha permitido determinar aquellas variables más relevantes que influyen en la adopción, uso e integración de las TD y que son tomadas como variables determinantes para la definición teórica de los perfiles de profesorado, estas variables son: la actitud y la autoeficacia mismas que son estudiadas con mayor frecuencia en la mayoría de las investigaciones.

Conforme a lo anterior se puede manifestar que las actitudes hacia el uso de las TD pueden definirse como sentimientos específicos que indican si una persona posee una percepción positiva o negativa hacia la adopción, uso e integración de las TD (Simpson et al., 1994), es así que las actitudes que el profesorado muestran hacia las tecnologías se pueden considerar como una variable determinante en relación con la integración de las mismas en el proceso educativo (Tondeur et al., 2018; Sang et al. , 2012)..

De la misma manera se ha demostrado en varios estudios que la autoeficacia del profesorado con respecto a la adopción uso e integración de las TD, esta positivamente correlacionada con la disposición del individuo a elegir y participar en actividades relacionadas con las TD (Scherer y Siddiq, 2015), con percepciones, emociones y capacidades de organizar y ejecutar las acciones necesarias para alcanzar los logros planteados con respecto a la integración de las TD (Compeau

Factores determinantes que permiten establecer tipologías del profesorado en el contexto de la innovación tecnológica educativa. B. Hidalgo-Cajo y M. Gisbert-Cervera. Página 7 de 23 
\& Higgins, 1995). De esta manera, se puede mencionar que el profesorado con mayores niveles de autoeficacia con respecto a las TD, son lo que hace uso de las mismas con mayor frecuencia (Siddiq et al., 2016).

El estudio de los perfiles de profesorado en función a la adopción, uso e integración de la TD podría aclarar las diferentes etapas que el profesorado cursa o se posiciona, para lo cual mediante el análisis de las características personales se genera una visión de las percepciones que tienen de los medios tecnológicos en contextos educativos.

A la hora de definir las tipologías o perfiles del profesorado hacia las TD, por medio de la revisión de la literatura se pudo identificar que las variables actitud y la autoeficacia percibida, son las variables más relevantes e influyentes que permiten ser utilizadas para la creación de perfiles del profesorado, cabe señalar que diversos estudios afirman que existe una correlación positiva entre estas variables (Bervell y Umar, 2017; Pamuk \& Peker, 2009; Abbitt \& Klett, 2007), por lo tanto el agrupamiento del profesorado con características similares lograra establecer las causas o factores más determinantes en el modo de adopción y uso de las TD en cada tipología.

A continuación, se puede observar los principales estudios que se han relacionado con la definición de perfiles del profesorado en el proceso de adopción uso e integración de las TD en el ámbito educativo.

Tabla 2.

Perfiles del profesorado en el proceso de integración de las TD en el proceso enseñanza aprendizaje

\begin{tabular}{|c|c|c|}
\hline Criterio & Perfiles & Referencia \\
\hline Creencias y practica tecnológica & $\begin{array}{l}\text { Entrada, Adopción, Adaptación, Apropiación, } \\
\text { Innovación }\end{array}$ & (Dwyer et al., 1991) \\
\hline Integración curricular de las TIC & $\begin{array}{l}\text { Dominio } \\
\text { Privilegiación } \\
\text { Reintegración } \\
\text { Apropiación } \\
\text { Internalización } \\
\end{array}$ & $\begin{array}{l}\text { (Colás Bravo et al., } \\
\text { 2005) }\end{array}$ \\
\hline Apropiación Tecnológica & $\begin{array}{l}\text { Acceso, } \\
\text { Capacitación y } \\
\text { apropiación }\end{array}$ & (Romani, 2008) \\
\hline $\begin{array}{l}\text { Actitud hacia la formación para el uso } \\
\text { de las TIC }\end{array}$ & $\begin{array}{l}\text { Entusiastas } \\
\text { Apocalípticos } \\
\text { Perdidos }\end{array}$ & $\begin{array}{l}\text { (Fernández Tilve \& } \\
\text { Álvarez Núñez, 2009) }\end{array}$ \\
\hline Adopción de las tecnologías & $\begin{array}{l}\text { Intrusear, Actitud, } \begin{array}{c}\text { Implementación, } \\
\text { apropiación, Integración curricular }\end{array}\end{array}$ & $\begin{array}{l}\text { (Reyes Gonzáles \& } \\
\text { Guevara Cruz, 2009) }\end{array}$ \\
\hline Competencia digital del profesorado & $\begin{array}{l}\text { Adopción (Destrezas digitales básicas) } \\
\text { Adaptación (Competencias Didácticas en TIC). } \\
\text { Apropiación (Estrategias de aprendizaje) } \\
\text { Innovación (Construcción digital) }\end{array}$ & (Krumsvik, 2009) \\
\hline Integración de las TIC. & $\begin{array}{l}\text { Tradicionalista centrado en el contenido } \\
\text { Adoptante selectivo Usuario inadvertido } \\
\text { Adaptadores creativos }\end{array}$ & (Donnelly et al., 2011) \\
\hline Actitud general hacia las tecnologías & $\begin{array}{l}\text { Docentes entusiasmados con las TIC Docentes } \\
\text { noveles en TIC }\end{array}$ & $\begin{array}{l}\text { (Gewerc \& Montero, } \\
\text { 2013) }\end{array}$ \\
\hline $\begin{array}{l}\text { Creencias, competencias digitales y } \\
\text { prácticas con TIC }\end{array}$ & $\begin{array}{l}\text { Inclusivos } \\
\text { Potenciales } \\
\text { Accidentales } \\
\text { Hostiles }\end{array}$ & $\begin{array}{l}\text { (Mama-Timotheou \& } \\
\text { Hennessy, 2013) }\end{array}$ \\
\hline $\begin{array}{l}\text { Fases de Clasificación de adoptantes } \\
\text { de blended learning en contextos } \\
\text { universitarios }\end{array}$ & $\begin{array}{l}\text { Fase de aprendizaje y conocimiento } \\
\text { Fase de persuasión - Actitud } \\
\text { Fase de decisión } \\
\text { Fase de aplicación inicial }\end{array}$ & $\begin{array}{l}\text { (Martin-Garcia et al., } \\
\text { 2014) }\end{array}$ \\
\hline
\end{tabular}

Factores determinantes que permiten establecer tipologías del profesorado en el contexto de la innovación tecnológica educativa. B. Hidalgo-Cajo y M. Gisbert-Cervera. Página 8 de 23 


\begin{tabular}{|c|c|c|}
\hline & $\begin{array}{l}\text { Fase de Integración y aplicación creativa a otros } \\
\text { contextos }\end{array}$ & \\
\hline $\begin{array}{l}\text { Actitudes, autoeficacia y competencia } \\
\text { digital docente }\end{array}$ & $\begin{array}{l}\text { Bajo perfil TIC } \\
\text { Alto perfil TIC }\end{array}$ & (Tondeur et al., 2019) \\
\hline $\begin{array}{lll}\text { Uso de } & \text { tecnologías } & \text { digitales } \\
\text { Confianza } & & \\
\text { Formación } & \text { específica } & \text { Acceso a } \\
\text { recursos } & & \\
\end{array}$ & $\begin{array}{l}\text { Activo } \\
\text { Seguro } \\
\text { Apoyado }\end{array}$ & $\begin{array}{l}\text { (European } \\
\text { Commission, 2019) }\end{array}$ \\
\hline
\end{tabular}

Dentro de esta perspectiva, la incorporación de las TD pasa necesariamente por las actitudes, creencias, formación y grado de aceptación que los docentes tengan hacia ellas (Broadbent, 2016; Tondeur et al., 2017), sin embargo la formación del profesorado está centrada desde una perspectiva tecno céntrica, lo que lleva a tener fuertes competencias tecnológicas - instrumentales para la incorporación de las TD, pero no tanto de carácter pedagógico (Prendes y Gutiérrez, 2013; Cabero y Barroso, 2016).

Se puede afirmar que los modelos sobre perfiles docentes ante la incorporación de las TD en el proceso enseñanza aprendizaje son escasos y no permiten visualizar la realidad del profesorado en las instituciones educativas, mismos que sostienen muy poca definición de las variables que influyen en este proceso, al no tomar en cuenta las necesidades y características del profesorado.

\section{Perfiles del profesorado hacia la adopción, uso e integración de las TD.}

Dado que el estudio se focaliza en el contexto del profesorado, el análisis toma en cuenta la importancia del desarrollo académico en la educación, a tal punto que es vital comprender e identificar las variables que definen e influye en la adopción, uso e integración de las TD en el proceso enseñanza aprendizaje, desde esta perspectiva se presenta que las actitudes como la autoeficacia hacia las TD son las variables que la investigación ha evidenciado como determinantes, como se muestra en la figura 2 .

En consecuencia, el profesorado es el principal responsable de la adopción, uso e integración de las TD en el proceso enseñanza aprendizaje (Agyei y Voogt, 2011), ciertamente aparte de los condicionantes sociales e institucionales de la adopción. Desde el contexto del profesorado se encuentra el proceso que cada profesor sigue al involucrarse por primera vez y permanecer en contacto con la TD, tomando en cuenta que las actitudes y la autoeficacia percibida hacia la labor académica, la propiedad y accesibilidad a la tecnología, la utilidad y facilidad de uso, la formación docente, la presión social y la importancia e interés que le brinde a las TD, son factores determinantes para un involucramiento personal con una permanencia relativamente estable.

Al referirse a las actitudes Jo Tondeur et al. (2016), considera que:

Las actitudes por parte del profesorado pueden definirse como una forma de sentir desde diferentes percepciones la adopción de las TD, esto trae en consecuencia la medición de las actitudes hacia las tecnologías considerándose como una evaluación en que el profesorado responde de forma favorable o desfavorable a la adopción, uso e integración de las TD, los factores como la utilidad, facilidad de uso percibido e interés son factores que ayudan a medir las actitudes del profesorado frente a las TD (p.12).

Gutiérrez (2014) considera que las actitudes del profesorado hacia las TD es un factor destacado que determina el proceso de cambio, al concebir la innovación como un estado mental o actitud. De este modo, las actitudes del profesorado determinaran en una fase inicial la predisposición hacia la utilización de nuevos modelos de enseñanza aprendizaje basados en la utilización o

Factores determinantes que permiten establecer tipologías del profesorado en el contexto de la innovación tecnológica educativa. B. Hidalgo-Cajo y M. Gisbert-Cervera. Página 9 de 23 
integración de las TD (Gewerc y Montero, 2013), por lo tanto las actitudes positivas o negativas que el profesorado tenga de las tecnologías, influyen en la adopción, uso e integración en el ámbito educativo, ya sea de forma individual como colaborativa con otros docentes. La investigación de Tondeur et al. (2016) afirmo que el uso de las TD en la educación se vio fuertemente afectado por las actitudes hacia las TD, por lo tanto, en este estudio se utiliza y se analiza un amplio espectro de dimensiones como "utilidad", "facilidad de uso", "interés" y "placer.", de la misma manera Sánchez-Mena et al. (2017) manifiestan que las variables, utilidad y facilidad de uso percibida influye de forma directa y positiva en la intención de los profesores de utilizar las TD, Njiku et al. (2019) hace referencia a la actitud del profesorado hacia las TD siendo la utilidad, la confianza, la ansiedad y la posición del profesorado, las variables más representativas hacia la integración de las TD, así también (G. A. Knezek et al., 2003; G. Knezek y Christensen, 2008, 2016; Morales, 2000; Agyei y Voogt, 2011), mencionan que para analizar las influencias de las actitudes del profesorado hacia las TD se debe examinar desde los factores como las competencias digitales, propiedad, acceso, y la enseñanza con TD.

De tal manera se puede mencionar que las actitudes hacia la tecnología influyen en la disposición de los profesores a aceptar la utilidad de la tecnología, así como en sus esfuerzos por integrarla en el proceso educativo (Hernández-Ramos et al., 2014). Sin embargo, a pesar del creciente acceso a la tecnología y, en algunos casos, de la formación de los profesores sobre la integración de la tecnología, se ha informado de una actitud negativa hacia ella, que podría ser la principal razón del bajo nivel de integración por parte de los profesores (Bozkurt \& Johnston-Wilder, 2011).

Al referirse a la variable autoeficacia Bandura (1986), afirma que es una parte integral de la creación de un cambio y aprendizaje exitoso como parte de la naturaleza humana, así también otros investigadores aseveran que es un factor predictor para el éxito académico (Richardson et al., 2012; Gewerc y Montero, 2013; Honicke y Broadbent, 2016; Birisci y Kul, 2019; Broadbent, 2016), desde el uso y las experiencias que el profesorado desarrolla por medio de las TD, lo que permite sentir autoconfianza en su integración, mediante la provisión, compromiso y colaboración docente en la aplicación práctica de la TD, creando un proceso eficaz de enseñanza aprendizaje. De la misma manera se puede mencionar que las creencias de autoeficacia de las TD pueden estimarse a partir de las actitudes del profesorado hacia las TD educativas.

En concreto, el estudio examina la manera que el profesorado pueda agruparse en función de sus actitudes y autoeficacia percibida hacia las TD, en el contexto del profesorado en su práctica docente.

Factores determinantes que permiten establecer tipologías del profesorado en el contexto de la innovación tecnológica educativa. B. Hidalgo-Cajo y M. Gisbert-Cervera. Página 10 de 23 


\section{Contexto del profesorado}

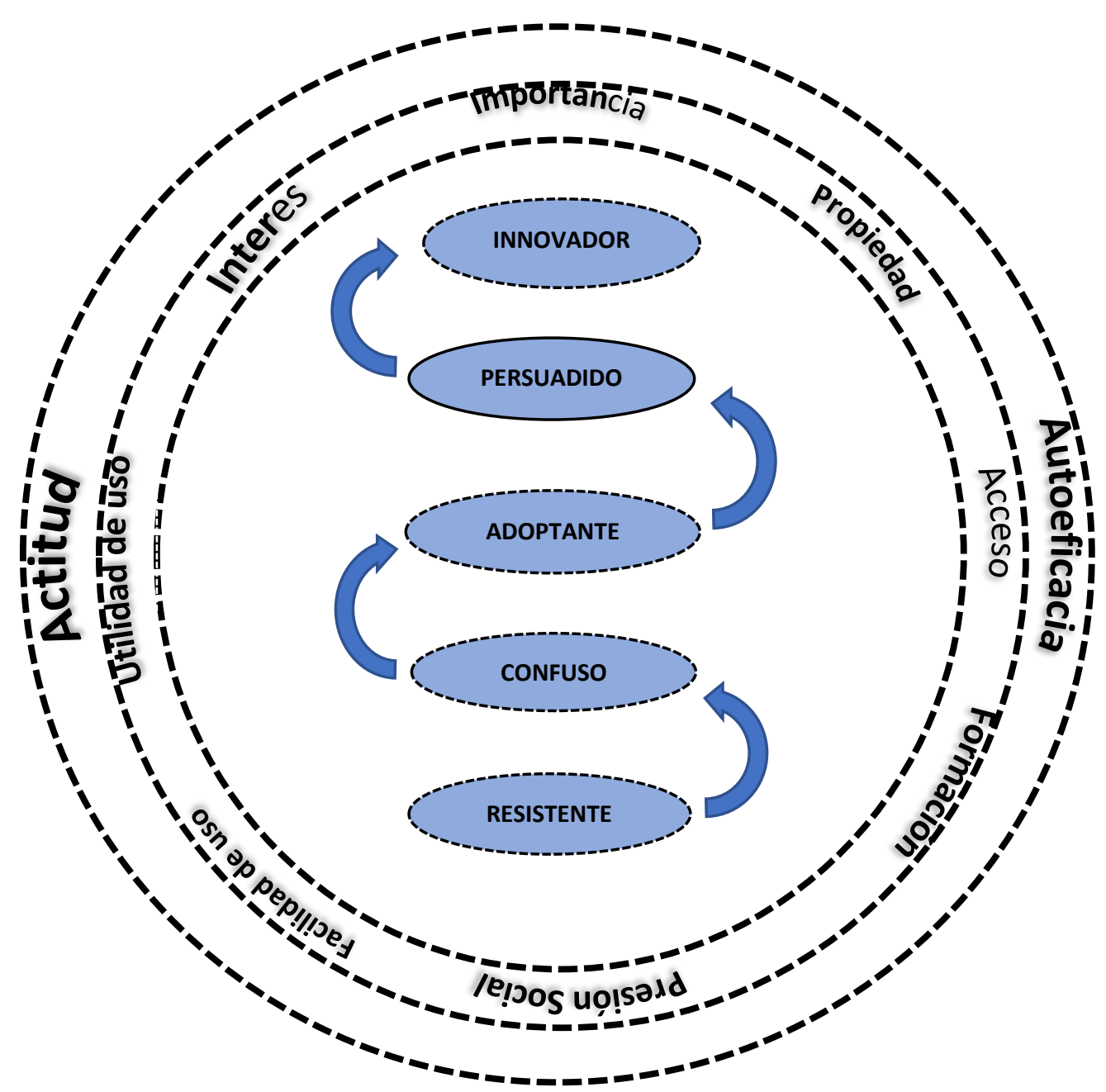

Figura 2. Perfiles docentes en cuanto a la integración de las TD desde el contexto del profesorado.

En lo referente a los perfiles del profesorado y según las variables y factores identificados, se logra generar un instrumento de análisis y de clasificación de los diferentes perfiles del profesorado, lo cual se puede distinguir cinco perfiles con respecto al uso educativo de las TD, según características individuales, variables y factores que permiten generar esta clasificación.

Por lo tanto, el estudio plantea los diferentes perfiles que el profesorado puede agruparse en dependencia de las variables autoeficacia y actitud del profesorado y los factores propiedad y accesibilidad a la tecnología, la utilidad y facilidad de uso, la formación docente, la presión social y la importancia e interés que le brinde a las TD.

1. Perfil resistente: se enmarcan en la resistencia a cambiar sus prácticas académicas mediadas por las TD, esto se debe al bajo interés que demuestra el profesorado ante la tecnología manifestadas en una limitada o baja propiedad y acceso a las mismas, esta actitud por parte del profesorado son analizados por Simin y Sani (2015) y Ocaña-Fernández et al. (2020), quienes mencionan que la propiedad y acceso a las TD influyen en el profesorado a tomar

Factores determinantes que permiten establecer tipologías del profesorado en el contexto de la innovación tecnológica educativa. B. Hidalgo-Cajo y M. Gisbert-Cervera. 
la decisión de adoptar la tecnología, de la misma manera Badia et al. (2016) y Petko (2012), aseguran que a medida que la TD esté disponible, conocida y valorada, su adopción y uso será evidente. En esta tipología el profesorado no tiene interés en la formación docente o autoformación en TD (Cózar et al., 2015), se mantienen en la desconfianza, brindan poca valoración e importancia a las TD en la práctica docente, a esto se suma la presión social que se somete el profesorado, ya que inicia un periodo de estrés por la influencia percibida debido a que, al aumentar el número de profesores que adoptan las TD, aumenta también la presión social, ante esta situación Jogezai et al. (2020), menciona que el profesorado experimenta un escenario de miedo y frustración a la hora de utilizar los recursos tecnológicos, debido a la falta de habilidades y competencias tecno-pedagógicas por parte de los profesores convirtiéndose en un problema significativo en este sentido, ya que el profesorado se desmotiva al no poder integrar las TD en su quehacer académico. Esta ineficacia de las competencias de los profesores surge también a nivel de comprensión de la utilidad y facilidad de uso de las TD con un conocimiento mínimo o casi nulo de la integración de las mismas como herramienta de aprendizaje para impartir sus clases, dado este escenario el profesorado se encuentra en una actitud negativa con una baja autoeficacia hacia el uso de las TD.

2. Perfil Confuso: se puede mencionar que el profesorado se encuentra en un dilema o incertidumbre ya que el cambio de resistente a confuso de una u otra forma en algún momento tenía que hacerlo, en esta etapa el profesorado se siente confundido dado que inicia el uso de la tecnología, sin encontrar el rumbo de como incorporarla en su práctica académica (Passey et al., 2018). El profesorado en este perfil brinda un marcado interés e importancia hacia las TD (Luan y Teo, 2009), por lo contrario conserva una baja propiedad o interés en poseer aparatos tecnológicos, el acceso a las TD sigue siendo baja dado que no logra identificar y aplicar su uso en el proceso educativo, en esta fase el profesorado se enmarca en formarse para comprender y tener una mejor percepción de las TD como herramienta didáctica, sin embargo aún no tiene claro la utilidad de su uso y aun requiere de mayor entrenamiento con las TD para poder determinar la facilidad de usar, la presión social sigue siendo alta dado que percibe que entre más se incrementa el número de adoptantes, también aumenta la influencia y la presión social sobre los resistentes y confusos en la adopción tecnológica, pero poco a poco, se ve envuelto por la innovación, esto cambia su actitud negativa hacia el uso de las TD, así también su autoeficacia cambia medianamente, dado que a mayor formación docente mayor autoeficacia hacia la integración de las TD (Al-Awidi \& Alghazo, 2012).

3. Perfil Adoptante: el profesorado en este nivel se encuentra en una fase de cambios y experimentación, ya que en él despierta el interés intrínseco como extrínseco de las TD, este interés provoca un cambio de actitud favorable tanto en la adquisición, propiedad y acceso a las TD, esta experiencia motiva al profesorado a la necesidad de la formación en TD, concientizándose en la importancia de conocer más sobre la aplicación y uso de la tecnología en los procesos educativos (Tadeu, 2020), convirtiéndose en un crítico en los diferentes procesos que experimenta, valorando la utilidad y facilidad de uso que brinda y representa las TD en el proceso educativo, sin embargo, la presión social persiste en un mediana densidad dado que él profesorado ya ha tomado la decisión de usar las TD, en este nivel el profesorado tiende a una marcada actitud positiva y de autoeficacia hacia las TD, señalando a estas como un aliado estratégico en el proceso enseñanza aprendizaje, lo que provoca una disminución de sus niveles de ansiedad y frustración redirigiendo esas energías hacia

Factores determinantes que permiten establecer tipologías del profesorado en el contexto de la innovación tecnológica educativa. B. Hidalgo-Cajo y M. Gisbert-Cervera. Página 12 de 23 
estrategias orientadas a mejorar y adecuar el proceso de enseñanza, con lo cual se garantiza el aumento de la productividad, tanto de profesorado como de estudiantado (Peinado et al., 2011). En este nivel el docente es propenso a aceptar la innovación.

4. Perfil persuadido: en esta fase la TD se integra completamente en la práctica académica, se logra la adopción, uso e integración de la tecnología por parte del profesorado, en esta tipología está convencido que la integración de las TD favorece al proceso enseñanza aprendizaje, por lo cual le brinda una alta importancia a las Tecnologías y hace mucho énfasis de su uso tanto dentro como fuera del aula de clase. Lo único que lo distancia del innovador es la propiedad y acceso a las TD, lo que puede implicar una menor apropiación de estas herramientas y de echo un menor uso, sin embargo, la presión social persiste en una mediana densidad ya que se siente más relajado y seguro con el proceso de adopción y uso de las tecnologías. Los persuadidos son quienes adoptan la innovación por primera vez, sin mucha discusión y análisis; pueden actuar como colaborador y jugar un papel importante para persuadir a otros actores de adoptar la innovación. En este nivel la actitud del profesorado es altamente positiva (Muhaimin et al., 2020; Admiraal et al., 2017), obteniendo una percepción alta sobre la tecnología, de la misma manera la autoeficacia percibida es alta ya que se ve envuelta en la formación docente, utilidad y facilidad de uso percibido sobre la aplicación de las TD en el proceso educativo.

5. Perfil Innovador: se caracteriza por tener una actitud y autoeficacia alta en el uso de las TD en el proceso educativo, el profesorado posee un alto interés e importancia a las mismas, este interés se ve demostrado en la propiedad y acceso a las TD por parte del profesorado, que le permite usar con frecuencia, desde esta perspectiva se puede mencionar que estos factores determinan o influyen la adopción, uso e integración de las TD. El profesorado en esta tipología percibe que su formación basada en la autoformación de las TD en el proceso educativo logra ser un profesor innovador que reconoce y aplica las TD y lo considera como un aliado estratégico en el proceso enseñanza aprendizaje, en esta tipología el profesorado tiende a una baja presión social, sin embargo se siente motivado para seguir manteniéndose en ese perfil, lo que se transforman en colaboradores que pueden motivar, liderar procesos de capacitación y promoción del uso de las TD a las demás tipologías.

Sin embargo se puede mencionar que estos perfiles planteados no son estáticos ya que un docente puede iniciar o adoptar un perfil de manera ordinal o nominal o a su vez puede llegar a adoptar diferentes perfiles, detalle que es corroborado por Donnelly et al. (2011) y Hao y Lee (2015).

La clave del modelo no está en aplicarlo en forma progresiva sino en idear cómo usar la tecnología para que proporcione al profesorado la oportunidad de enseñar en otros escenarios, imposibles de imaginar sin ella.

\section{Conclusiones y discusión de resultados}

Los recursos educativos digitales, nos ofrecen un abanico de posibilidades que pueden apoyar a la transformación de la educación. En este sentido, debemos partir de una perspectiva integral del cambio educativo basado en la tecnología para comprender sus efectos. En este caso, el estudio ha servido para evaluar uno de los elementos más relevantes y menos estudiados en la literatura sobre la incorporación de las TD en el aula por parte del profesorado.

Factores determinantes que permiten establecer tipologías del profesorado en el contexto de la innovación tecnológica educativa. B. Hidalgo-Cajo y M. Gisbert-Cervera. Página 13 de 23 
Las TD han adquirido tal relevancia que es impensable la enseñanza sin su participación, pero es necesario también conocer como el profesorado se apropia de la tecnología en el proceso enseñanza aprendizaje a partir de determinadas variables que con mayor frecuencia son aplicadas en los estudios de este tipo, con sus diferentes factores que ayudan a identificar las tipologías del profesorado o perfiles de apropiación de las TD de las cuales se espera que el profesorado vaya atravesando hacia la integración en función de la apropiación tecnológica.

Por otro lado las TD han generado una transformación en todas las áreas y más aún en el ambiente educativo donde el profesorado, como elemento clave en el proceso integrador de las TD, debe ser analizado e identificado para lograr la innovación educativa, por lo cual el estudio logra identificar las variables más relevantes en el contexto del profesorado como son la Actitud y Autoeficacia del profesorado hacia las TD, estas variables identificadas son las que influyen significativamente en la adopción, uso y apropiación de la tecnología.

Desde esta perspectiva el estudio analiza las características del profesorado en torno a estas dos variables localizando, desprendiéndose diversos factores que permite agrupar al profesorado en referencia a la apropiación de las TD, siendo estas la Utilidad de uso, Propiedad, acceso a las TD, Formación docente, Facilidad de uso, Importancia, presión social e Interés ante las TD.

El estudio en base a las variables y factores identificados concluye planteando un modelo que determina la tipología del profesorado universitario con cinco niveles: resistente, confundido, adoptante, persuadido e innovador, así como mencionan varios estudios que no hay un único nivel, sino que se encuentran una diversidad de ellos (Krumsvik, 2009; Dwyer et al., 1991). El modelo planteado define cada una de las diferentes fases de adopción, uso y apropiación de las $\mathrm{TD}$, hasta llegar al nivel en el que los docentes son capaces de integrar la tecnología en el proceso enseñanza aprendizaje y generar innovación, sin embargo, se debe tomar en cuenta que cada docente puede encontrase atravesando por distintas dimensiones de manera única y de ubicarse en cualquier perfil planteado.

Tabla 3.

Resumen de perfiles docentes en función de las variables

\begin{tabular}{|c|l|c|c|c|c|c|}
\hline \multicolumn{2}{|c|}{ Variables /Factores } & $\begin{array}{c}\text { Perfil 1. } \\
\text { Resistente }\end{array}$ & $\begin{array}{c}\text { Perfil 2. } \\
\text { Confuso }\end{array}$ & $\begin{array}{c}\text { Perfil 3. } \\
\text { Adoptante }\end{array}$ & $\begin{array}{c}\text { Perfil 4. } \\
\text { Persuadido }\end{array}$ & $\begin{array}{c}\text { Perfil 5. } \\
\text { Innovador }\end{array}$ \\
\hline $\mathbf{1}$ & Actitud & & & & & \\
\hline $\mathbf{2}$ & Autoeficacia & & & & & \\
\hline 3 & Utilidad de uso & & & & & \\
\hline 4 & Propiedad & & & & \\
\hline 5 & Acceso a las TD & & & & & \\
\hline $\mathbf{6}$ & Formación docente & & & & & \\
\hline 7 & Facilidad de uso & & & & & \\
\hline $\mathbf{8}$ & Importancia de las TD & & & & & \\
\hline 9 & Presión social & & & & & \\
\hline $\mathbf{1 0}$ & Interés ante las TD & & & & & \\
\hline
\end{tabular}

Nota: La tabla muestra el nivel de valoración de las variables en cada perfil en función a los colores planteados: Rojo: Bajo; Amarillo: Medio; y Verde: Alto.

La revisión de la literatura, en el contexto de la adopción, uso e integración de la TD, en el contexto del profesorado se ve afectada por diversas variables y factores. La integración de las TD en la enseñanza y el aprendizaje, como revela la literatura, es un proceso continuo (Koehler y Mishra, 2009), y el papel de los profesores sigue siendo más central en este sentido (Jamieson, 2013). Todas las investigaciones anteriores como indican Hassan et al. (2013) y Kisirkoi (2015) por ejemplo, destacan que la disponibilidad y la accesibilidad a las TD son muy imprescindible

Factores determinantes que permiten establecer tipologías del profesorado en el contexto de la innovación tecnológica educativa. B. Hidalgo-Cajo y M. Gisbert-Cervera. 
para que los profesores puedan utilizarlos en el proceso enseñanza y aprendizaje, sin embargo considerar sólo la provisión de las TD no puede asegurar su integración, sino la asociación de varias factores que logren este objetivo.

En este sentido, parece que aún queda camino por recorrer en el logro de una integración efectiva de las TD por parte del profesorado en el proceso enseñanza aprendizaje.

Presentación del artículo: 01 de octubre 2021

Fecha de aprobación: 14 de enero de 2022

Fecha de publicación: 30 de enero de 2022

Hidalgo-Cajo, B. \& Gisbert-Cervera, M. (2022) Factores determinantes que permiten establecer tipologías del profesorado en el contexto de la innovación tecnológica educativa. RED. Revista Educación a Distancia, 22(69).

http://dx.doi.org/10.6018/red.499171

\section{Financiación}

Este trabajo no ha recibido ninguna subvención específica de los organismos de financiación en los sectores públicos, comerciales o sin fines de lucro

\section{Referencias bibliográficas}

Abbitt, J. T., \& Klett, M. D. (2007). Identifying influences on attitudes and self-efficacy beliefs towards technology integration among pre-service educators. Electronic Journal for the Integration of Technology in Education, 6(1), 28-42.

Admiraal, W., Louws, M., Lockhorst, D., Paas, T., Buynsters, M., Cviko, A., Janssen, C., de Jonge, M., Nouwens, S., Post, L., van der Ven, F., \& Kester, L. (2017). Teachers in school-based technology innovations: A typology of their beliefs on teaching and technology. Computers and Education, 114, 57-68. https://doi.org/10.1016/j.compedu.2017.06.013

Aguilar-Flores, S. M., \& Chiang-Vega, M. M. (2020). Factores que determinan el uso de las TIC en adultos mayores de Chile. Revista Científica, 39(3), 296-308. https://doi.org/10.14483/23448350.16054

Agyei, D. D., \& Voogt, J. M. (2011). Exploring the potential of the will, skill, tool model in Ghana: Predicting prospective and practicing teachers' use of technology. Computers and Education, 56(1), 91-100. https://doi.org/10.1016/j.compedu.2010.08.017

Al-Awidi, H. M., \& Alghazo, I. M. (2012). The effect of student teaching experience on preservice elementary teachers' self-efficacy beliefs for technology integration in the UAE. Educational Technology Research and Development, 60(5), 923-941. https://doi.org/10.1007/s11423-012-9239-4

Araiza, M., \& Pedraza, E. (2019). Discernimiento de los docentes por género en el uso de las

Factores determinantes que permiten establecer tipologías del profesorado en el contexto de la innovación tecnológica educativa. B. Hidalgo-Cajo y M. Gisbert-Cervera. Página 15 de 23 
TIC en el aula a partir de las competencias digitales. Espacios, 4O(21), 21-28.

Babić, S., Sučić, S. K., \& Sinković, G. (2020). Understanding the Factors that Influence Secondary School Teachers' Intention to Use e-Learning Technologies for Teaching After the COVID-19 Pandemic. 2020 43rd International Convention on Information, Communication and Electronic Technology (MIPRO), 848-853. https://doi.org/10.23919/MIPRO48935.2020.9245433

Badia, A., Campos, L. C., Vargas d'Uniam, J., \& Díaz, G. S. (2016). La percepción de la utilidad de la tecnología conforma su uso para enseñar y aprender. Revista Electronica de Investigacion Educativa, 18(3), 92-104. http://www.scielo.org.mx/pdf/redie/v18n3/16074041-redie-18-03-00095.pdf

Badia, A., \& Iglesias, S. (2019). The Science Teacher Identity and the Use of Technology in the Classroom. Journal of Science Education and Technology, 28(5), 532-541. https://doi.org/10.1007/s10956-019-09784-w

Bandura, A. (1986). Social foundations of thought and action. Englewood Cliffs, NJ, 1986.

Bervell, B., \& Umar, I. N. (2017). A decade of LMS acceptance and adoption research in SubSahara African higher education: A systematic review of models, methodologies, milestones and main challenges. Eurasia Journal of Mathematics, Science and Technology Education, 13(11), 7269-7286. https://doi.org/10.12973/ejmste/79444

Bin, E., Islam, A. Y. M. A., Gu, X., Spector, J. M., \& Wang, F. L. (2020). A study of Chinese technical and vocational college teachers' adoption and gratification in new technologies. British Journal of Educational Technology, O(0). https://doi.org/10.1111/bjet.12915

Birisci, S., \& Kul, U. (2019). Predictors of technology integration self-efficacy beliefs of preservice teachers. Contemporary Educational Technology, 10(1), 75-93. https://doi.org/10.30935/cet.512537

Bozkurt, G., \& Johnston-Wilder, S. (2011). Factors Influencing Student Teachers' Use of ICT in Mathematics Teaching. In S.-M. Barton, J. Hedberg, \& K. Suzuki (Eds.), Proceedings of Global Learn 2011 (pp. 627-634). Association for the Advancement of Computing in Education (AACE). https://www.learntechlib.org/p/37237

Broadbent, J. (2016). Academic success is about self-efficacy rather than frequency of use of the learning management system. Australasian Journal of Educational Technology, 32(4), 38-49. https://doi.org/10.14742/ajet.2634

Cabero, J., \& Barroso, J. (2016). ICT teacher training: a view of the TPACK model. Cultura $y$ Educación, 28(3), 633-663. https://doi.org/10.1080/11356405.2016.1203526

Christensen, R., Eichhorn, K., Prestridge, S., Petko, D., Sligte, H., Baker, R., Alayyar, G., \& Knezek, G. (2018). Supporting Learning Leaders for the Effective Integration of Technology into Schools. Technology, Knowledge and Learning, 23(3), 457-472. https://doi.org/10.1007/s10758-018-9385-9

Colás Bravo, P., Rodríguez López, M., \& Jiménez Cortés, R. (2005). Evaluación de e-learning. Indicadores de calidad desde el enfoque sociocultural.e. Teoria de La Educación, Educacion y Cultura En La Sociedad de La Informacion. https://campus.usal.es/ teoriaeducacion/rev_numero_06_2/n6_02_art_colas_rodriguez_ji menez.htm

Compeau, D. R., \& Higgins, C. A. (1995). Application of Social Cognitive Theory to Training for Computer Skills. 6(2), 118-143.

Factores determinantes que permiten establecer tipologías del profesorado en el contexto de la innovación tecnológica educativa. B. Hidalgo-Cajo y M. Gisbert-Cervera. Página 16 de 23 
Cózar, R., del Valle, M., Hernández, J. A., \& Hernández, J. R. (2015). Uso de la realidad aumentada en la formacion inicial de maestros. Digital Education, O(27), 138-153. http://revistes.ub.edu/index.php/der/article/view/11622/pdf

Davies, S., Mullan, J., \& Feldman, P. (2017). Rebooting learning for the digital age: What next for technology-enhanced higher education? HEPI Report, 93, 46. http://www.hepi.ac.uk/wp-content/uploads/2017/02/Hepi_Rebooting-learning-for-thedigital-age-Report-93-02_02_17Web.pdf

Donnelly, D., McGarr, O., \& O'Reilly, J. (2011). A framework for teachers' integration of ICT into their classroom practice. Computers and Education, 57(2), 1469-1483. https://doi.org/10.1016/j.compedu.2011.02.014

Drossel, K., Eickelmann, B., \& Gerick, J. (2017). Predictors of teachers' use of ICT in school the relevance of school characteristics, teachers' attitudes and teacher collaboration. Education and Information Technologies, 22(2), 551-573. https://doi.org/10.1007/s10639016-9476-y

Dwyer, D., Ringstaff, C., \& Sandholtz, J. H. (1991). Changes in teachers' beliefs and practices in technology-rich classrooms. Educational Leadership, 48(8), 45-52. http://coe.nevada.edu/nstrudler/ACOTMay91.pdf

Ertmer, P. A., \& Ottenbreit-Leftwich, A. T. (2010). Teacher Technology Change. Journal of Research on Technology in Education, 42(3), 255-284. https://doi.org/10.1080/15391523.2010.10782551

European Commission. (2019). 2nd Survey of schools: ICT in education - Objective 1: Benchmarking progress in ICT in schools. https://doi.org/10.2759/23401

Farjat-aguilar, A. A., \& Barroso-tanoira, F. G. (2009). Percepción y actitud de los profesores sobre el uso de tec-nologías de la información y la comunicación en el proceso enseñanza-aprendizaje . Un estudio de caso. 7-14.

Fernández-Márquez, E., Leiva-Olivencia, J., \& López-Meneses, E. (2018). Competencias digitales en docentes de Educación Superior. Competencias Digitales En Docentes de Educación Superior, 12(1), 213-231. https://doi.org/https://doi.org/10.19083/ridu.12.558

Fernández Tilve, M., \& Álvarez Núñez, Q. (2009). Un estudio de caso sobre un proyecto de innovación con TIC en un centro educativo de Galicia: ¿acción o reflexión? Bordón. Revista de Pedagogía, 61(1), 95-108.

Garcia, C., Yot, C., Rodriguez, E., \& Zorrilla-Salgador, J. P. (2020). Factores Determinantes Del Uso De Dispositivos Móviles En La Formación Inicial Docente De Uruguay. Educação Em Revista, 36. https://doi.org/10.1590/0102-4698216356

Gewerc, A., \& Montero, L. (2013). La integración de las TIC en las instituciones educativas. 69. https://doi.org/10.4438/1988-592X-RE-2011-362-163

Gil-Flores, J., Rodríguez-Santero, J., \& Torres-Gordillo, J. J. (2017). Factors that explain the use of ICT in secondary-education classrooms: The role of teacher characteristics and school infrastructure. Computers in Human Behavior, 68, 441-449. https://doi.org/10.1016/j.chb.2016.11.057

Goh, E., \& Sigala, M. (2020). Integrating Information \& Communication Technologies (ICT) into classroom instruction: teaching tips for hospitality educators from a diffusion of innovation approach. Journal of Teaching in Travel and Tourism, 20(2), 156-165. https://doi.org/10.1080/15313220.2020.1740636

Factores determinantes que permiten establecer tipologías del profesorado en el contexto de la innovación tecnológica educativa. B. Hidalgo-Cajo y M. Gisbert-Cervera. Página 17 de 23 
Gutiérrez Porlán, I. (2014). Perfil del profesor universitario español en torno a las competencias en tecnologías de la información y la comunicación. Pixel-Bit Revista de Medios y Educación, 51-65. https://doi.org/10.12795/pixelbit.2014.i44.04

Hall, A. B., \& Trespalacios, J. (2019). Personalized Professional Learning and Teacher SelfEfficacy for Integrating Technology in K-12 Classrooms. Journal of Digital Learning in Teacher Education, 35(4), 221-235. https://doi.org/10.1080/21532974.2019.1647579

Hao, Y., \& Lee, K. S. (2015). Teachers' concern about integrating Web 2.0 technologies and its relationship with teacher characteristics. Computers in Human Behavior, 48, 1-8. https://doi.org/10.1016/j.chb.2015.01.028

Hassan, T., \& Rahim Sajid, A. (2013). ICTs in learning: Problems faced by Pakistan. Journal of Research \& Reflections in Education (JRRE), 7(1), 52-64.

Hernández-Ramos, J. P., Martínez-Abad, F., García Peñalvo, F. J., Esperanza Herrera García, M., \& Rodríguez-Conde, M. J. (2014). Teachers' attitude regarding the use of ICT. A factor reliability and validity study. Computers in Human Behavior, 31(1), 509-516. https://doi.org/10.1016/j.chb.2013.04.039

Hidalgo-Cajo, B. G., \& Gisbert-Cervera, M. (2021). Diseño y validación de un instrumento para evaluar la adopción de la tecnología digital en el profesorado universitario. Campus Virtuales , Revista Cientifica de Tecnología Educatíva, 10, 51-67.

http://www.uajournals.com/campusvirtuales/journal/19/3.pdf

Hidalgo Cajo, B. G., Hidalgo Cajo, D. P., \& Hidalgo Cajo, I. M. (2017). El impacto de las redes sociales como herramientas de comunicación, interacción y colaboración en el proceso enseñanza aprendizaje en la educación superior. SATHIRI, 12(104-113).

http://revistasdigitales.upec.edu.ec/index.php/sathiri/article/view/56/96

Hixon, E., \& Buckenmeyer, J. (2009). Revisiting technology integration in Schools: Implications for professional development. Computers in the Schools, 26(2), 130-146. https://doi.org/10.1080/07380560902906070

Holland, D. D., \& Piper, R. T. (2016). A technology integration education (TIE) model for millennial preservice teachers: Exploring the canonical correlation relationships among attitudes, subjective norms, perceived behavioral controls, motivation, and technological, pedagogical, and content . Journal of Research on Technology in Education, 48(3), 212226. https://doi.org/10.1080/15391523.2016.1172448

Holmes, B., Gardner, J., \& Galanouli, D. (2007). Striking the right chord and sustaining successful professional development in information and communications technologies. Journal of In-Service Education, 33(4), 389-404. https://doi.org/10.1080/13674580701687799

Honicke, T., \& Broadbent, J. (2016). The influence of academic self-efficacy on academic performance: A systematic review. Educational Research Review, 17, 63-84. https://doi.org/10.1016/j.edurev.2015.11.002

Hu, X., Gong, Y., Lai, C., \& Leung, F. K. S. (2018). The relationship between ICT and student literacy in mathematics, reading, and science across 44 countries: A multilevel analysis. Computers and Education, 125, 1-13. https://doi.org/10.1016/j.compedu.2018.05.021

Jo Tondeur, Sarah Van de Velde, Hans Vermeersch, \& Mieke Van Houtte. (2016). Gender Differences in the ICT Profile of University Students: A Quantitative Analysis. DiGeSt. Journal of Diversity and Gender Studies, 3(1), 57. https://doi.org/10.11116/jdivegendstud.3.1.0057

Factores determinantes que permiten establecer tipologías del profesorado en el contexto de la innovación tecnológica educativa. B. Hidalgo-Cajo y M. Gisbert-Cervera. Página 18 de 23 
Jogezai, N. A., Baloch, F. A., \& Ismail, S. A. M. M. (2020). Hindering and enabling factors towards ICT integration in schools: A developing country perspective. Elementary Education Online, 19(3), 1537-1547. https://doi.org/10.17051/ilkonline.2020.733176

Kirkwood, A., \& Price, L. (2014). Technology-enhanced learning and teaching in higher education: what is "enhanced" and how do we know? A critical literature review. Learning, Media and Technology, 39(1), 6-36. https://doi.org/10.1080/17439884.2013.770404

Kisirkoi, F. K. (2015). Integration of ICT in Education in a Secondary School in Kenya: A Case Study. Literacy Information and Computer Education Journal, 6(2), 1904-1909. https://doi.org/10.20533/licej.2040.2589.2015.0253

Knezek, G. A., Christensen, R., \& Fluke, R. (2003). Testing a will, skill, tool model of technology integration. American Educational Research Association, 4, 1-12. http://www.eric.ed.gov/PDFS/ED475762.pdf

Knezek, G., \& Christensen, R. (2008). the Importance of Information Technology Attitudes and Competencies. Computing, 321-331.

Knezek, G., \& Christensen, R. (2016). Extending the will, skill, tool model of technology integration: adding pedagogy as a new model construct. Journal of Computing in Higher Education, 28(3), 307-325. https://doi.org/10.1007/s12528-016-9120-2

Koehler, M. J., \& Mishra, P. (2009). What is technological pedagogical content knowledge? Contemporary Issues in Technology and Teacher Education. Education \& Information Technology Library, 9(1), 60-70. http://www.tpck.org/.

Krumsvik, R. (2009). Situated learning in the network society and the digitised school. European Journal of Teacher Education, 32(2), 167-185. https://doi.org/10.1080/02619760802457224

Li, L. (2010). A critical review of technology acceptance literature. Southwest Decisino Sciences Institute, 22. http://www.swdsi.org/swdsi2010/SW2010_Preceedings/papers/PA104.pdf

Li, L. Y., \& Tsai, C. C. (2017). Accessing online learning material: Quantitative behavior patterns and their effects on motivation and learning performance. Computers and Education, 114, 286-297. https://doi.org/10.1016/j.compedu.2017.07.007

Luan, W. S., \& Teo, T. (2009). Investigating the Technology Acceptance among Student Teachers in Malaysia: An Application of the Technology Acceptance Model (TAM). The Asia-Pacific Education Researcher, 18(2). https://doi.org/10.3860/taper.v18i2.1327

Mama-Timotheou, M., \& Hennessy, S. (2013). Developing a typology of teacher beliefs and practices concerning classroom use of ICT. Computers and Education, 68, 380-387. https://doi.org/10.1016/j.compedu.2013.05.022

Marín-Díaz, V., Riquelme, I., \& Cabero-Almenara, J. (2020). Uses of ICT tools from the perspective of chilean university teachers. Sustainability (Switzerland), 12(15), 1-12. https://doi.org/10.3390/su12156134

Martin-Garcia, A. V, Hernandez-Serrano, J., \& Sánchez Gómez, M. C. (2014). Fases y clasificación de adoptantes de blended learning En Contextos Universitarios . 4, 457-477.

Mercader, C. (2019). Las resistencias del profesorado universitario a la utilización de las tecnologías digitales. Aula Abierta, 48(2), 167.

Factores determinantes que permiten establecer tipologías del profesorado en el contexto de la innovación tecnológica educativa. B. Hidalgo-Cajo y M. Gisbert-Cervera. Página 19 de 23 
https://doi.org/10.17811/rifie.48.2.2019.167-174

Mirzajani, H., Mahmud, R., Mohd, A., \& Su-Luan, W. (2015). A Review of Research Literature on Obstacles that Prevent Use of ICT in Pre-Service Teachers' Educational Courses. International Journal of Education and Literacy Studies, 3(2). https://doi.org/10.7575/aiac.ijels.v.3n.2p.25

Morales Arce, V. (2013). Desarrollo de competencias digitales docentes en la educación básica. Apertura: Revista de Innovación Educativa, 5(1), 88-97.

Morales, C. (2000). Etapas de adopción de la tecnología informática al salón de clases. 1-11.

Mosquera-González, D., Valencia-Arias, A., Benjumea-Arias, M., \& Palacios-Moya, L. (2021). Factors associated with using information and communication technologies (ICT) in the learning processes of engineering students. Formacion Universitaria, 14(2), 121-132. https://doi.org/10.4067/S0718-50062021000200121

Muhaimin, A., Habibi, A., Mukminin, A., \& Hadisaputra, P. (2020). Science teachers' integration of digital resources in education: A survey in rural areas of one Indonesian province. Heliyon, 6(8), e04631. https://doi.org/10.1016/j.heliyon.2020.e04631

Naik, G., Chitre, C., Bhalla, M., \& Rajan, J. (2020). Impact of use of technology on student learning outcomes: Evidence from a large-scale experiment in India. World Development, 127, 104736. https://doi.org/10.1016/j.worlddev.2019.104736

Ndlovu, M., Ramdhany, V., Spangenberg, E. D., \& Govender, R. (2020). Preservice teachers' beliefs and intentions about integrating mathematics teaching and learning ICTs in their classrooms. ZDM - Mathematics Education, 52(7), 1365-1380. https://doi.org/10.1007/s11858-020-01186-2

Njiku, J., Maniraho, J. F., \& Mutarutinya, V. (2019). Understanding teachers' attitude towards computer technology integration in education: A review of literature. Education and Information Technologies, 24(5), 3041-3052. https://doi.org/10.1007/s10639-019-09917-z

Ocaña-Fernández, Y., Valenzuela-Fernández, L., \& Morillo-Flores, J. (2020). La competencia digital en el docente universitario. Propósitos y Representaciones, 8(1). https://doi.org/10.20511/pyr2020.v8n1.455

Okoli, C., \& Schabram, K. (2010). A guide to conducting a systematic literature review of information systems research. Sprouts. Concordia University, Canada.

Palos-Sanchez, P., Reyes-Menendez, A., \& Saura, J. R. (2019). Models of adoption of information technology and cloud computing in organizations. Informacion Tecnologica, 30(3), 3-12. https://doi.org/10.4067/S0718-07642019000300003

Pamuk, S., \& Peker, D. (2009). Turkish pre-service science and mathematics teachers' computer related self-efficacies, attitudes, and the relationship between these variables. Computers and Education, 53(2), 454-461. https://doi.org/10.1016/j.compedu.2009.03.004

Passey, D., Shonfeld, M., Appleby, L., Judge, M., Saito, T., \& Smits, A. (2018). Digital Agency: Empowering Equity in and through Education. Technology, Knowledge and Learning, 23(3), 425-439. https://doi.org/10.1007/s10758-018-9384-x

Peinado, S., Bolívar, J. M., \& Briceño, L. A. (2011). Actitud hacia el uso de computadoras de docentes. 7(1), 86-105.

Pérez García, E. A., \& Andrade Cázares, R. A. (2020). Orientación de la competencia digital del profesor universitario en las propuestas de integración de TIC. IE Revista de Investigación

Factores determinantes que permiten establecer tipologías del profesorado en el contexto de la innovación tecnológica educativa. B. Hidalgo-Cajo y M. Gisbert-Cervera. Página 20 de 23 
Educativa de La REDIECH, 11, e905. https://doi.org/10.33010/ie_rie_rediech.v11i0.905

Pérez, Ó. M. M. G., Almenara, J. C., \& Sampedro, B. S. (2016). Valoraciones de la "Aceptación de la Tecnología de Formación Virtual" por profesores universitarios asistentes a un curso de formación virtual. Edutec. Revista Electrónica de Tecnología Educativa, O(56).

Petko, D. (2012). Teachers' pedagogical beliefs and their use of digital media in classrooms: Sharpening the focus of the "will, skill, tool" model and integrating teachers" constructivist orientations. Computers and Education, 58(4), 1351-1359. https://doi.org/10.1016/j.compedu.2011.12.013

Prendes Espinosa, M. P., \& Gutiérrez Porlán, I. (2013). Competencias tecnológicas del profesorado en las universidades españolas. Revista de Educacion, 361, 196-222. https://doi.org/10.4438/1988-592X-RE-2011-361-140

Reyes Gonzáles, D. S., \& Guevara Cruz, H. (2009). Adopción de las Tecnologías infocomunicacionales (TI) en docentes: Actualizando Enfoques. Revista Electrónica Teoría de La Educación. Educación y Cultura En La Sociedad de La Información, 10(1). https://www.redalyc.org/html/2010/201018023008/

Richardson, M., Abraham, C., \& Bond, R. (2012). Psychological correlates of university students' academic performance: A systematic review and meta-analysis. Psychological Bulletin, 138(2), 353-387. https://doi.org/10.1037/a0026838

Rodriguez, A., Garcia, E., \& Ibañez. (2009). Las TIC en la educación superior. 8, 1-18.

Rolf, E., Knutsson, O., \& Ramberg, R. (2019). An analysis of digital competence as expressed in design patterns for technology use in teaching. British Journal of Educational Technology, 50(6), 3361-3375. https://doi.org/10.1111/bjet.12739

Romani, C. (2008). Aprendizaje Adaptable y Apropiación Tecnológica: Reflexiones prospectivas. 3er Encuentro de Auto-Estudio de Las Universidades Públlicas Mexicanas.

Sánchez-Mena, A., Martí-Parreño, J., \& Aldás-Manzano, J. (2017). The effect of age on teachers' intention to use educational video games: A TAM approach. Electronic Journal of E-Learning, 15(4), 355-366. https://files.eric.ed.gov/fulltext/EJ1154704.pdf

Sang, G., Valcke, M., van Braak, J., Zhu, C., Tondeur, J., \& Yu, K. (2012). Challenging science teachers' beliefs and practices through a video-case-based intervention in China's primary schools. Asia-Pacific Journal of Teacher Education, 40(4), 363-378. https://doi.org/10.1080/1359866X.2012.724655

Scherer, R., \& Siddiq, F. (2015). Revisiting teachers' computer self-efficacy: A differentiated view on gender differences. Computers in Human Behavior, 53, 48-57. https://doi.org/10.1016/j.chb.2015.06.038

Selcen Guzey, S., \& Roehrig, G. H. (2012). Integrating Educational Technology into the Secondary Science Teaching. Contemporary Issues in Technology and Teacher Education, 12(2), 162-183.

Siddiq, F., Scherer, R., \& Tondeur, J. (2016). Teachers' emphasis on developing students' digital information and communication skills (TEDDICS): A new construct in 21st century education. Computers and Education, 92-93, 1-14.

https://doi.org/10.1016/j.compedu.2015.10.006

Simin, G., \& Sani, I. M. (2015). Effectiveness of ICT integration in Malaysian schools: A quantitative analysis. International Research Journal for Quality in Education, 2(8), 1-12.

Factores determinantes que permiten establecer tipologías del profesorado en el contexto de la innovación tecnológica educativa. B. Hidalgo-Cajo y M. Gisbert-Cervera. Página 21 de 23 
Simpson, R. D., Koballa, T. R., Oliver, J. S., \& Crawley, F. E. (1994). Research on the affective dimension of science learning. Handbook of Research on Science Teaching and Learning, $1,211-234$.

Sung, Y. T., Chang, K. E., \& Liu, T. C. (2016). The effects of integrating mobile devices with teaching and learning on students' learning performance: A meta-analysis and research synthesis. Computers and Education, 94, 252-275.

https://doi.org/10.1016/j.compedu.2015.11.008

Tadeu, P. (2020). La competencia científico-tecnológica en la formación del futuro docente: algunos aspectos de la autopercepción en respeto a la integración de las TIC en el aula. Educatio Siglo XXI, 38(3 Nov-Feb), 37-54. https://doi.org/10.6018/educatio.413821

Tafazoli, D., Parra, M. E. G., \& Abril, C. A. H. (2019). Attitude towards computer-assisted language learning: Do gender, age and educational level matter? Teaching English with Technology, 19(3), 22-39.

Teo, T. (2011). Factors influencing teachers' intention to use technology: Model development and test. Computers and Education, 57(4), 2432-2440.

https://doi.org/10.1016/j.compedu.2011.06.008

Tondeur, J., Aesaert, K., Prestridge, S., \& Consuegra, E. (2018). A multilevel analysis of what matters in the training of pre-service teacher's ICT competencies. Computers and Education, 122, 32-42. https://doi.org/10.1016/j.compedu.2018.03.002

Tondeur, J., Pareja Roblin, N., van Braak, J., Voogt, J., \& Prestridge, S. (2017). Preparing beginning teachers for technology integration in education: ready for take-off?

Technology, Pedagogy and Education, 26(2), 157-177. https://doi.org/10.1080/1475939X.2016.1193556

Tondeur, J., Scherer, R., Baran, E., Siddiq, F., Valtonen, T., \& Sointu, E. (2019). Teacher educators as gatekeepers: Preparing the next generation of teachers for technology integration in education. British Journal of Educational Technology, 50(3), 1189-1209. https://doi.org/10.1111/bjet.12748

Tondeur, J., van Braak, J., Ertmer, P. A., \& Ottenbreit-Leftwich, A. (2017). Understanding the relationship between teachers' pedagogical beliefs and technology use in education: a systematic review of qualitative evidence. Educational Technology Research and Development, 65(3), 555-575. https://doi.org/10.1007/s11423-016-9481-2

Trujillo-Torres, J. M., Hossein-Mohand, H., Gómez-García, M., Hossein-Mohand, H., \& Cáceres-Reche, M. P. (2020). Mathematics teachers' perceptions of the introduction of ict: The relationship between motivation and use in the teaching function. Mathematics, 8(12), 1-17. https://doi.org/10.3390/math8122158

Ursavaş, Ö. F., Yalçın, Y., \& Bakır, E. (2019). The effect of subjective norms on preservice and in-service teachers' behavioural intentions to use technology: A multigroup multimodel study. British Journal of Educational Technology, 50(5), 2501-2519. https://doi.org/10.1111/bjet.12834

Valdés-Cuervo, A., Angulo Armenta, J., Nieblas Valencia, E., Lucero Zambrano, F., \& Arreola Olivarría, C. (2009). Actitudes de docentes de secundaria hacia el uso de las TIC. In Investigación Educativa (Issue 11, pp. 60-76).

Wen, K. Y. K., \& Hua, T. K. (2020). Esl teachers' intention in adopting online educational technologies during covid-19 pandemic. Journal of Education and E-Learning Research, 7(4), 387-394. https://doi.org/10.20448/journal.509.2020.74.387.394

Factores determinantes que permiten establecer tipologías del profesorado en el contexto de la innovación tecnológica educativa. B. Hidalgo-Cajo y M. Gisbert-Cervera. Página 22 de 23 
Wu, B., Yu, X., \& Hu, Y. (2019). How does principal e-leadership affect ICT transformation across different school stages in K-12 education: Perspectives from teachers in Shanghai. British Journal of Educational Technology, 50(3), 1210-1225. https://doi.org/10.1111/bjet.12766

Zempoalteca Durán, B., Barragán Lopez, J. F., González Martinez, J., \& Guzmán Flores, T. (2017). Formación en TIC y competencia digital en la docencia en instituciones públicas de educación superior. Apertura, 9(1), 80-96. https://doi.org/10.18381/Ap.v9n1.922 